\title{
The Effect of Reloading on Disuse Muscle Atrophy: Time Course of Hypertrophy and Regeneration Focus- ing on the Myofiber Cross-sectional Area and Myonu- clear Change
}

\author{
Kazumi ZUSHI $^{1,2}$ and Toshiaki YAMAZAKI ${ }^{1}$ \\ 1) Division of Health Sciences, Graduate School of Medical Science, Kanazawa University, 5-11-80 Kodatsuno, Kanazawa, Ishikawa \\ 920-0942, Japan \\ 2) Department of Rehabilitation, Minamigaoka Hospital
}

\begin{abstract}
The purpose of this study was to investigate the effect of reloading on atrophied muscle and the time course of hypertrophy and regeneration. Forty-nine male Wistar rats were randomly assigned to groups for hindlimb suspension (HS), hindlimb suspension and reloading (R), or control (C0). Rats in the HS group were suspended for 14 days. Rats in the $R$ group were randomly divided into five subgroups for different post-hindlimb-suspension recovery times. Briefly, each subgroup was suspended for 14 days and given 1 day of reloading (R1), 3 days of reloading (R3), 7 days of reloading (R7), 10 days of reloading (R10), or 14 days of reloading (R14). Myonuclear numbers were significantly decreased in the groups with hindlimb suspension and 1 day and 3 days of reloading compared with that in the control group. We focused on the processes of change of mean myofiber cross-sectional area and myonuclear domain size; the degrees of increase of both indexes were limited until 3 days of reloading, and significantly increased after 7 days of reloading. An important finding of the current study was that the processes of muscle hypertrophy and regeneration did not show uniform change. In addition, there were differences in the ratio of increase among the stages of hypertrophy and regeneration. Therefore, consideration of the duration and method of physiotherapeutic intervention for atrophied muscle on the basis of the process of hypertrophy and regeneration is needed to provide more effective physiotherapy.

Key words: hindlimb suspension, reloading, time course, myofiber cross-sectional area, myonuclear
\end{abstract}

(J Jpn Phys Ther Assoc 15: 1-8, 2012)

D isuse syndrome is a secondary disorder that is caused by decreased activity due to long-term bed rest or disease. It involves degeneration of the cardiovascular system and atrophy of the musculoskeletal system ${ }^{1)}$. It is also a subject of rehabilitation. Disuse muscle atrophy causes decline of movement function. In addition, disuse muscle atrophy may lead to a vicious circle involving a decline in daily activity after cure of the original disease. Therefore, inhibition of disuse muscle atrophy and promotion of hypertrophy and regeneration of atrophied muscle as soon as possible

Received: August 1, 2011

Accepted: January 5, 2012

Advance Publication by J-STAGE: March 27, 2012

Correspondence to: Kazumi Zushi, Division of Health Sciences, Graduate School of Medical Science, Kanazawa University, 5-11-80 Kodatsuno, Kanazawa, Ishikawa 920-0942, Japan.

e-mail: yamazaki@mhs.mp.kanazawa-u.ac.jp are important. Many animal experimental studies have reported that weight-bearing stimuli ${ }^{2}$ or stretching stimuli ${ }^{3,4)}$ inhibit muscle atrophy.

However, in a clinical context, a therapeutic procedure has rarely been applied during the progression of muscle atrophy. In many cases, therapeutic procedures are started after the progression of muscle atrophy to a certain degree. Myofiber has reversibility in terms of response to change of mechanical stimuli. Atrophied muscle could be hypertrophied and regenerated by an increase of mechanical stimuli. Indeed, some studies have histologically examined the duration of such an approach that is necessary for the regeneration of atrophied muscle. Mitchell et al. ${ }^{5)}$ showed that decrease of type I myofiber cross-sectional area (MCSA) by 14 days of hindlimb suspension was recovered to the control level by 14 days of reloading. 
Skeletal muscle is a complex tissue composed of multinucleate myofibers. Myofibers are structured in cytoplasm, and are surrounded and interspaced by myonuclei. The theoretical amount of cytoplasm controlled by each myonucleus is called the DNA unit size or the myonuclear domain size $(\mathrm{MND})^{6-10)}$. If MND is controlled perfectly by myonuclei, MCSA and myonuclei change in parallel upon hindlimb suspension or reloading. Changes of myonuclear number and MND with muscle atrophy have been researched using various muscle atrophy models. It has been stated that hindlimb suspension model could reproduce long-term bed rest $^{11)}$. In this model, both myonuclear number and MND are reduced ${ }^{7,12)}$.

Some studies on the effects of reloading on atrophied muscle have been reported. Previous study has shown that reduction in type I myonuclear number by 14 days of hindlimb suspension is recovered to the control level by 14 days of reloading ${ }^{5}$. Another study has reported that 16 days of reloading following 16 days of hindlimb suspension recovered myonuclear number, but to a level that was still less than that of age-matched control ${ }^{7}$. On the other hand, 16 days of reloading recovered MND to the level in agematched control after its reduction by 16 days of hindlimb suspension ${ }^{7}$. Mozdiziak et al. ${ }^{10)}$ reported that both 2 weeks and 9 weeks of reloading following 28 days of hindlimb suspension recovered myonuclear number and MND from the decreases caused by 28 days of hindlimb suspension. However, neither myonuclear number nor MND reached the age-matched control level. These results suggested that reductions of myonuclear number and MND accompanying muscle atrophy were recovered by reloading. Furthermore, none of these studies examined the time-course changes of MCSA, myonuclear number, and MND. It is necessary to study the time course of hypertrophy and regeneration after hindlimb suspension in order to determine the effectiveness and efficiency of physical therapy accurately. In addition, for considering the concept of MND, MCSA was structured by several MND, and MND were controlled by each myonucleus. Therefore, there is a good reason for studying myonuclear number and MND.

Inoue $e t$ al. ${ }^{13)}$ showed the possibility that vulnerability of myofiber was induced by disuse muscle atrophy. In addition, they reported not only that myofiber necrosis occurred by muscle atrophy but also that the initiation of reloading injured weak atrophied myofiber. It was possible that full bodyweight-bearing immediately after reloading overloaded vulnerable muscle. Accordingly, studying how myofiber necrosis induced by reloading influences muscle hypertrophy and how regeneration occurs might be key for decision-making regarding appropriate loading for atrophied muscle.

Therefore, the purpose of this study was to investigate the effect of reloading on atrophied muscle and the time course of hypertrophy and regeneration by analyzing the following: 1) myonuclear number and MND, and 2) MCSA histogram in the early stage of reloading of atrophied rat soleus muscle. In addition, we investigated whether full bodyweight-bearing overloaded atrophied muscle by analyzing myofiber necrosis and how regeneration occurred by analyzing the myofibers with centrally located myonuclei from a pathological perspective.

\section{Methods}

\section{Materials}

Forty-nine male Wistar rats aged 8 weeks were used in this study. All rats were housed in individual cages under a 12-hour light-dark cycle.

\section{Protocol}

All protocols were approved by the committee on animal experimentation of Kanazawa University (No. AP091350).

The rats were randomly assigned to groups for hindlimb suspension (HS, $n=7$ ), hindlimb suspension and reloading ( $R, n=35)$, or control $(\mathrm{C} 0, \mathrm{n}=7$ ). Hindlimb suspension was based on the procedure described in a previous report ${ }^{14)}$. Rats could move freely by using their forelimbs to obtain food and water ad libitum. Rats in the HS group were suspended for 14 days. Rats in the $\mathrm{R}$ group were randomly divided into five subgroups for different post-hindlimbsuspension recovery times. Briefly, each subgroup was suspended for 14 days and given 1 day of reloading (R1: $\mathrm{n}=7$ ), 3 days of reloading (R3: $\mathrm{n}=7$ ), 7 days of reloading $(R 7: n=7), 10$ days of reloading (R10: $n=7)$, or 14 days of reloading (R14: $n=7)$. Rats in the R groups were loaded with the full bodyweight and allowed to pursue activities in the cage freely. We set the pre-experiment status $(\mathrm{C} 0: \mathrm{n}=$ 7) as a control group.

\section{Muscle preparation and histochemical analysis}

After the experimental period, bodyweights were measured and the right soleus muscles were removed under anesthesia. After soleus muscle wet weights were measured, muscles were rapidly frozen in liquid nitrogen-cooled isopentane and stored at $-70^{\circ} \mathrm{C}$ until analysis. In order to undertake histological analysis, $6-\mu \mathrm{m}$ and $8-\mu \mathrm{m}$ frozen sections were cut from the central part of the right soleus muscle using a cryostat (Sakura Finetek CRY03, Tokyo, Japan). To measure MCSA and to count the number of myonuclei, muscle sections of $6 \mu \mathrm{m}$ were reacted with dystrophin antibody and 4',6-diamidino-2-phenylindole (DAPI). Briefly, the sections were air-dried and then fixed with $4 \%$ paraformaldehyde for $15 \mathrm{~min}$ at room temperature. The sections were washed in $0.1 \mathrm{M}$ PBS and incubated with $0.1 \mathrm{M}$ PBS with $10 \%$ normal goat serum and $1 \%$ Triton $\mathrm{X}-100$ at room temperature to block nonspecific staining. The sections were again washed in PBS and incubated with 
Table 1. Muscle wet weight, myofiber cross sectional area, myonuclear number and myonuclear domain size (Mean \pm SD)

\begin{tabular}{lccccccc}
\hline Groups & C0 & HS & R1 & R3 & R7 & R10 & R14 \\
\hline MW $(\mathrm{mg})$ & $90.3 \pm 4.0$ & $71.9 \pm 8.8$ & $69.1 \pm 8.3^{*}$ & $90.1 \pm 14.0^{+}$ & $102.3 \pm 8.6^{\#+}$ & $112.1 \pm 21.1^{\#+}$ & $96.7 \pm 11.1^{\#+}$ \\
MCSA $\left(\mu \mathrm{m}^{2}\right)$ & $2180 \pm 590$ & $1418 \pm 710$ & $1518 \pm 590$ & $1441 \pm 634$ & $1987 \pm 730$ & $2200 \pm 893$ & $2354 \pm 1025$ \\
MN & $1.02 \pm 0.12$ & $0.83 \pm 0.08^{*}$ & $0.85 \pm 0.04^{*}$ & $0.84 \pm 0.13^{*}$ & $0.88 \pm 0.09$ & $0.90 \pm 0.11$ & $0.91 \pm 0.09$ \\
MND $\left(\mu \mathrm{m}^{2}\right)$ & $2099 \pm 368$ & $1760 \pm 404^{\S}$ & $1855 \pm 500^{\S}$ & $1846 \pm 437^{\S}$ & $2363 \pm 639$ & $2521 \pm 351$ & $2611 \pm 276$ \\
\hline
\end{tabular}

MCSA: myofiber cross sectional area. There were significant differences between each experiment group except between control and 10 days of reloading, hindlimb suspension and 3 days of reloading, 1day and 3 days of reloading group.

${ }^{\#} \mathrm{P}<0.05$ when compared to HS of MW. ${ }^{+} \mathrm{P}<0.05$ when compared to R1 of MW.

$* \mathrm{P}<0.05$ when compared to $\mathrm{C} 0$ of $\mathrm{MW}$ and $\mathrm{MN} .{ }^{\S} \mathrm{P}<0.05$ when compared to R14 of MND.

MW: muscle wet weight. MN: myonuclear number. MND: myonuclear domain size.

monoclonal anti-dystrophin antibody (D8043, SIGMA) diluted 1:3000 with $0.1 \mathrm{M}$ PBS with 5\% normal goat serum and $0.3 \%$ Triton $\mathrm{X}-100$ for 48 hours at $4{ }^{\circ} \mathrm{C}$. After washing in PBS, they were incubated with the secondary Alexa fluor 594 goat anti-mouse IgG (Invitrogen Japan) diluted 1:400 with $0.1 \mathrm{M}$ PBS with 5\% normal goat serum and $0.3 \%$ Triton $\mathrm{X}-100$ overnight at $4{ }^{\circ} \mathrm{C}$. They were washed again in PBS and incubated with 4',6-diamidino-2-phenylindole (DAPI; SIGMA). We measured MCSA using image analysis software Image $\mathrm{J}$ targeting more than 100 myofibers in the center and the periphery of each sample. Nuclei within the dystrophin-positive sarcolemma were counted in more than 100 myofibers in each sample as myonuclei. Secondly, sections of $8 \mu \mathrm{m}$ were stained with standard hematoxylin and eosin (HE) for counts of myofiber necrosis and myofibers with centrally located nuclei, targeting more than 2500 myofibers in each group. Myofiber necrosis in this study was defined as infiltration of phagocytes or remarkable reduction in staining ${ }^{13,15)}$.

\section{The methods of calculating each index}

Myonuclear numbers in each myofiber were calculated by dividing total counted myonuclear number of myofibers by total myofiber number (myonuclear number $=$ total myonuclear number/total myofiber number). MND were calculated by dividing total MCSA of myofiber by total myonuclear number of myofiber $(\mathrm{MND}=$ total $\mathrm{MCSA} /$ total myonuclear number). The proportions of myofiber necrosis and myofibers with centrally located nuclei were calculated by dividing total myofiber necrosis and myofibers with centrally located nuclei by total myofiber number in each group, respectively.

\section{Statistical analysis}

Statistical analysis involved one-way analysis of variance (ANOVA) for MCSA, myonuclear number, and MND. In the case of significant differences, Bonferroni multiple comparison test was applied. To test for statistically significant differences, $\chi^{2}$ test and Bonferroni correction were used for the proportions of myofiber necrosis and myofibers with centrally located nuclei. Measured values are expressed as mean \pm standard deviation (SD) and statistical significance was assumed at $\mathrm{p}<0.05$.

\section{Results}

\section{Muscle wet weight and myofiber cross-sectional area}

The results of muscle wet weights are described in Table 1. Reloading stimuli increased muscle wet weights. There were significant differences between hindlimb suspension and 7 days, 10 days, and 14 days of reloading. In addition, the value in the reloading group at 1 day was significantly less than those at 3 days, 7 days, 10 days, and 14 days in the reloading group.

Compared with that in the control group, MCSA was decreased significantly by 14 days of hindlimb suspension (Table 1). Reloading induced gradual recovery of MCSA, with the exception that MCSA decreased slightly at 3 days in the reloading group compared with that at 1 day in the reloading group. There were significant differences between each experiment group except between control and 10 days of reloading, hindlimb suspension and 3 days of reloading, and 1 day and 3 days of reloading.

According to the MCSA histogram, hindlimb suspension generally shifted the histogram to the left, and reloading recovered MCSA and shifted the histogram to the right (Fig. 1). Moreover, in the control, hindlimb suspension, 1 day, 3 days, and 7 days of reloading groups, the number of myofibers tended to increase gradually to the number of myofibers having the mean MCSA. On the other hand, groups with 10 days and 14 days of reloading initially tended to show increased myofibers with a small area $\left(\sim 400 \mu \mathrm{m}^{2}\right)$, and next showed a decrease of this number, and then finally showed a gradual increase of the number of myofibers with the mean MCSA.

\section{Myonuclear number and myonuclear domain size}

Myonuclear numbers were significantly decreased in 

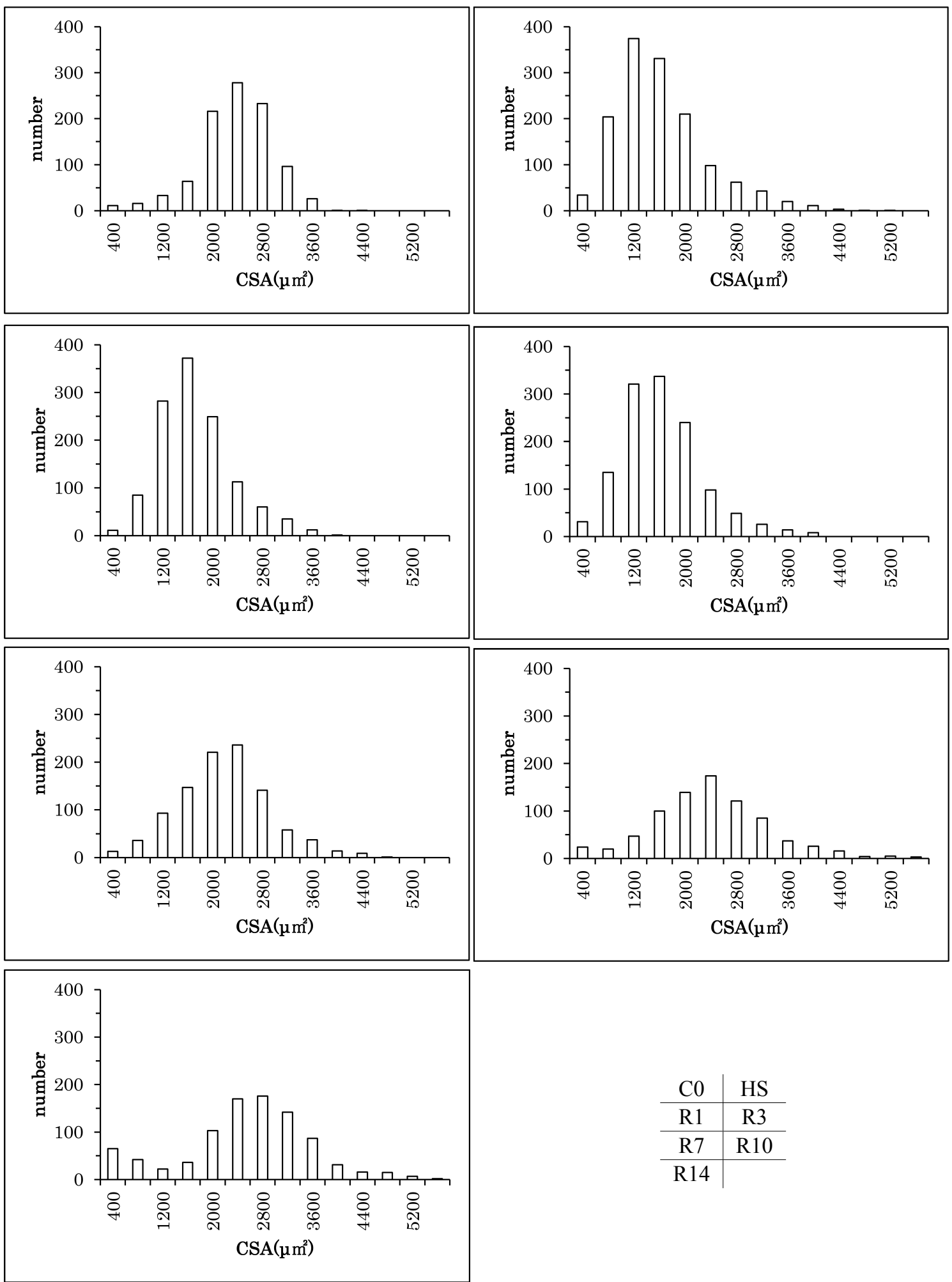

\begin{tabular}{c|c} 
C0 & HS \\
\hline R1 & R3 \\
\hline R7 & R10 \\
\hline R14 &
\end{tabular}

Fig. 1. Histogram of myofiber cross-sectional area

The vertical line indicates the number of myofiber.

The horizontal axis indicates myofiber cross-sectional area that is divided by every $400 \mu \mathrm{m}^{2}$. 


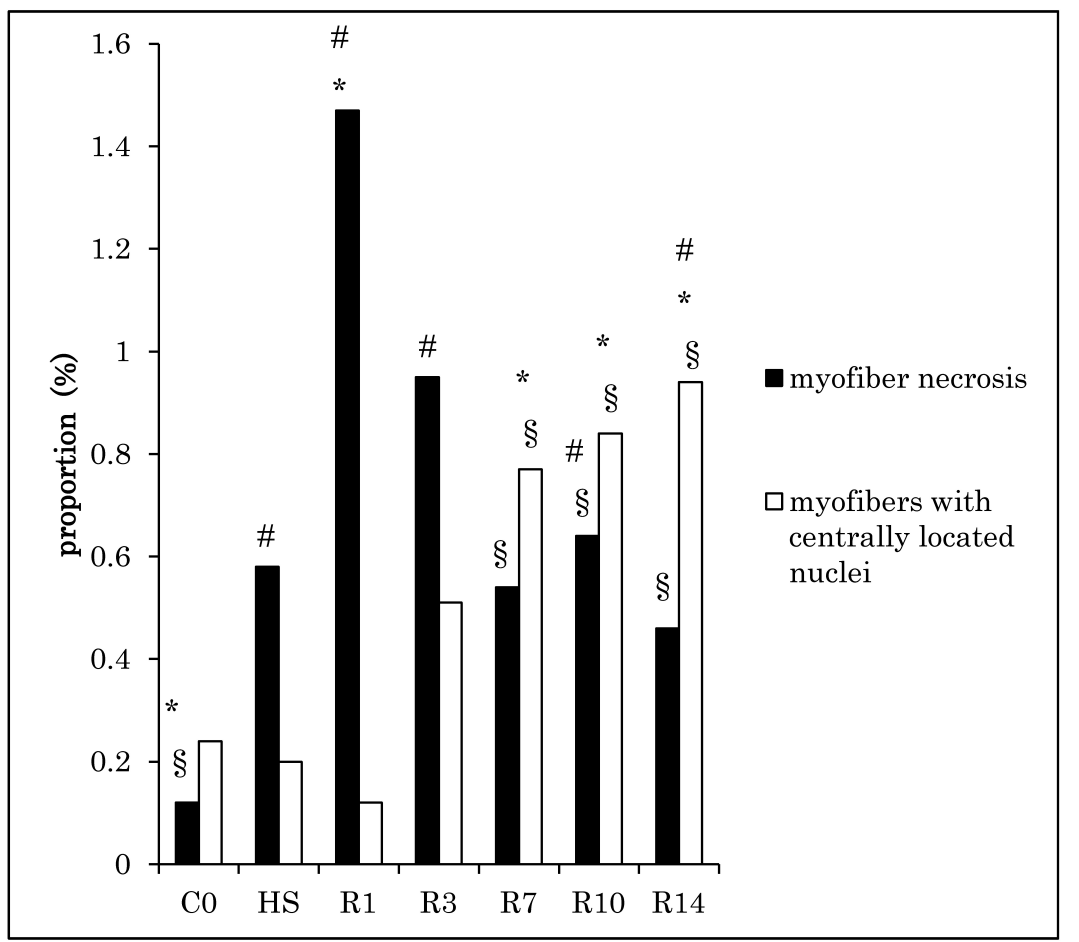

Fig. 2. The proportions of myofiber necrosis and myofibers with centrally located nuclei ${ }^{\#} \mathrm{P}<0.05$ when compared to $\mathrm{C} 0 .{ }^{*} \mathrm{P}<0.05$ when compared to HS. ${ }^{\S} \mathrm{P}<0.05$ when compared to R1.

the groups with hindlimb suspension and 1 day and 3 days of reloading compared with that in the control group (Table 1). Groups with 7 days, 10 days, and 14 days of reloading still had fewer myonuclei than the control group, but there were no significant differences. Moreover, there were no reloading groups that showed a significant recovery compared with the hindlimb suspension group.

MND tended to decrease upon 14 days of hindlimb suspension, but no significant differences were found (Table 1). All reloading groups tended to show a gradual increase compared with the hindlimb suspension group. There were significant differences between the hindlimb suspension group, 1 day of reloading group, 3 days of reloading group, and 14 days of reloading group.

\section{The proportions of myofiber necrosis and myofibers with centrally located nuclei}

The proportions of myofiber necrosis in the control group and the hindlimb suspension group were $0.12 \%$ and $0.58 \%$, respectively, which were significantly different (Fig. 2). Moreover, the proportion of myofiber necrosis in the 1 day of reloading group was $1.47 \%$, which was significantly greater than that in the hindlimb suspension group. In the 3 days of reloading group, the proportion of myofiber necrosis was $0.95 \%$. The proportion of myofiber necrosis was highest in the 1 day of reloading group. Thereafter, it tended to decrease. There were significant differences between the 1 day of reloading group and the 7 days, 10 days, and 14 days of reloading groups. The proportions of myo- fiber necrosis in hindlimb suspension, 1 day, 3 days, and 10 days of reloading groups were significantly higher than that of the control. In contrast, the proportion of myofibers with centrally located nuclei was gradually increased by reloading. In the 1 day of reloading group, the proportion of myofibers with centrally located nuclei was $0.12 \%$, which was increased to $0.94 \%$ in the 14 days of reloading group. There were significant differences between hindlimb suspension, 1 day of reloading and 7 days, 10 days, and 14 days of reloading groups. The proportion of myofibers with centrally located nuclei in the 14 days of reloading group was significantly higher than that in the control.

\section{Discussion}

Mean MCSA was decreased by hindlimb suspension and recovered by reloading. MCSA was recovered to that of the control by 10 days of reloading; namely, that in the 10 days of reloading group showed no significant difference compared with that in the control group, while that in the 14 days of reloading group was significantly greater than that in the control group. Previous study ${ }^{5}$ showed that decrease of type I MCSA by 14 days of hindlimb suspension was recovered to the control level by 14 days of reloading. There was a difference in this study in terms of the recovery. The reason for this difference might be related to a difference of materials. Mitchell et al. ${ }^{5)}$ used mice in their study, but we used rat soleus muscle in this study. In addition, because we used the pre-experiment group as a control group, there is a 
possibility that our 'recovery' involved growth.

Because myofiber has reversibility in terms of response to change of mechanical stimuli, MCSA of atrophied muscle is hypertrophied by increase of mechanical stimuli. Skeletal muscle is composed of some myonuclei and MND. Therefore, MCSA atrophy and hypertrophy accompany decrease and increase of myonuclear number and MND. Myonuclear number was significantly decreased by 14 days of hindlimb suspension. Previous study ${ }^{16}$ reported that 14 days of hindlimb suspension significantly increased myonuclear apoptosis. Another study) reported that 16 days of hindlimb suspension significantly decreased myonuclear number. The results of this study were supported by previous studies.

In terms of the effect of reloading on myonuclear number, the values in the 1 day and 3 days of reloading groups were still significantly lower than that in the control group, but 7 days, 10 days, and 14 days of reloading groups did not show significant differences from the control. However, on and after 7 days of reloading, there were no significant differences with the hindlimb suspension group. Therefore, myonuclear number did not increase at an early stage of reloading, but subsequently gradually increased to the control level. This trend of increase might involve recovery and growth, although 14 days of reloading were not sufficient for recovery to the pre-experiment level. Previous study showed that myonuclear number was not significantly increased by 2 days of reloading following 16 days of unloading ${ }^{7}$, and that 14 days of reloading following 14 days of unloading significantly recovered myonuclear number to the control level compared with unloading ${ }^{5)}$. In the present study, myonuclear number was not significantly increased by 14 days of reloading, but tended to recover to the control level. Therefore, the results of this study were similar to those of previous studies.

MND tended to be decreased by 14 days of hindlimb suspension, but no significant difference was found. However, the atrophy of rat soleus myofiber induced by 14 days of hindlimb suspension led to a $35 \%$ decrease in MCSA and a $19 \%$ decrease in myonuclear number; the data indicated that the amount of fiber atrophy was greater than the loss of myonuclei. Thus, MND might decrease upon hindlimb suspension. Moreover, Wang et al. ${ }^{7}$ stated that hindlimb suspension may lead to not only a decrease in the number of myonuclei but also a reduction of their function. Because 16 days of unloading increased the mean size of myonuclei but left the total DNA content in a single myonucleus unchanged, the DNA concentration in a myonucleus was lower in the muscle fibers of unloaded rats than in control rats. Therefore, in this study, it is thought that there were no significant differences but that MND might be decreased by unloading.

MND tended to increase gradually by reloading. MND was significantly increased by 14 days of reloading com- pared with that in the 14 days of hindlimb suspension group. In addition, MND value of the 14 days of reloading group was greater than that of the control group, but there was no significant difference. A previous study ${ }^{10}$ showed that MND was increased accompanied by growth, so the increase of MND in this study was accompanied by growth and recovered from its decrease caused by atrophy.

In this study, myofiber necrosis significantly emerged upon 14 days of hindlimb suspension compared with that in the control; thus, the vulnerability of muscle was induced by disuse muscle atrophy. In addition, the beginning of reloading increased myofiber necrosis significantly. Nguyen et al. ${ }^{17)}$ stated that 24 hours of reloading injured atrophied muscle, and the extent of damage was $1.5 \%$. In the present study, the proportion of myofiber necrosis was $1.47 \%$ upon 1 day of reloading. The results of this study are similar to those of previous reports. The proportion of myofiber necrosis was highest upon 1 day of reloading and then this value decreased markedly. In addition, the proportion of myofiber necrosis was significant decreased on and after 7 days of reloading. It was stated that $\mathrm{ED} 1^{+}$macrophages removed fragments produced by myofiber necrosis ${ }^{18}$. Furthermore, the infiltration of $\mathrm{ED} 1^{+}$macrophages was highest at 2 days of reloading and decreased rapidly thereafter ${ }^{19}$. In this study, myofiber necrosis was identified by the infiltration of macrophages into myofiber or significantly lower staining of myofiber. Therefore, it is possible that myofiber necrosis was significantly increased by 1 day of reloading, and later declined.

Regeneration of myofiber necrosis was started by activation of satellite cells and fusion to existing myofiber as a source of new myonuclei ${ }^{15}$. At 5 or 7 days from myofiber necrosis, regenerated myofiber was recognized morphologically as myofiber with a small diameter and a large myonucleus ${ }^{15}$. In addition, regenerated myofiber had a centrally located nucleus. Therefore, we focused on this type of myofiber as regenerated myofiber. According to the histogram of MCSA, 10 days and 14 days of reloading groups had more myofiber of small diameter $\left(\sim 400 \mu \mathrm{m}^{2}\right)$ than the control, hindlimb suspension, 1 day, 3 days, and 7 days of reloading groups. The difference of the shape of the histogram was considered to be due to the fact that atrophied myofibers were hypertrophied by reloading, so MCSA was increased as a whole, but regeneration of myofiber necrosis generated myofibers of small diameter. On the other hand, the proportion of myofibers with centrally located nuclei was $0.1 \sim 0.2 \%$ in the control, hindlimb suspension, and 1 day of reloading groups, and this proportion was increased by reloading.

Tidball et al. ${ }^{20)}$ showed that the proportion of myofibers with centrally located nuclei upon 4 days of reloading was more than five times as much as that upon 2 days of reloading. In this study, the proportion of myofibers with centrally located nuclei upon 3 days of reloading was almost four 
times, and upon 7 days of reloading was almost six times, as much as upon 1 day of reloading, and the value was $0.94 \%$ in the 14 days of reloading group. On and after 7 days of reloading, the proportions of myofibers with centrally located nuclei were significantly higher than in the hindlimb suspension and 1 day of reloading groups. The centrally located nuclei were seen not only with the regeneration of myofiber but also with the differentiation of new myonuclei accompanied by activation of satellite cells in the hypertrophic region. Therefore, there was a possibility of a difference in the duration of increase between myofibers with a small diameter and myofibers with centrally located nuclei. Moreover, according to the finding that the increase of myofibers with centrally located nuclei occurred earlier than that of myofibers with small diameter, it is possible that a hypertrophic reaction occurred significantly more than regeneration of myofiber necrosis immediately after reloading. In addition, according to the finding that there were many myofibers with small diameter upon 10 days and 14 days of reloading, it is possible that regeneration of myofiber necrosis started immediately after reloading, but regeneration reaction gradually became active throughout the period of reloading. However, in this study, we did not analyze satellite cell activity, an index of hypertrophy and regeneration; therefore, in order to clarify this supposition, we should investigate the activation of satellite cells.

We focused on the processes of change of mean MCSA and MND; the degrees of recovery of both indexes were limited until 3 days of reloading, and significantly recovered after 7 days of reloading. The time point of 7 days was when myonuclear number was recovered by reloading; namely, at this point, no significant difference from the control group was observed. The results of this study suggested that the increase of mechanical stimuli induced myofiber hypertrophy and regeneration because myofibers with centrally located nuclei tended to increase at the early stage of reloading. In addition, recovery of myonuclear number influenced the increase of MCSA and MND, and might increase the efficiency of muscle hypertrophy. Allen et $a l{ }^{12)}$ reported that decrease of myonuclear number led to decreased total DNA content, which accompanied decreased MCSA. In contrast, increase of myonuclei induced by reloading in this study contributed to an increase of total DNA content, and thus it may be related to an increase of MCSA. Furthermore, previous study ${ }^{7}$ showed that an increase of myonuclear size and a decrease of DNA concentration remained significant upon 2 days of reloading, but recovered to the control levels upon 16 days of reloading. It is possible that the function of existing myonuclei was gradually improved by reloading and the development of muscle hypertrophy became more efficient.

The results of this study indicated the possibility that the change in the myonuclear number with reloading stimuli affected hypertrophy of MCSA, and that MND recov- ered to the control level earlier than myonuclear number. Moreover, we suggested the possibility that the distribution of MCSA was influenced by muscle hypertrophy rather than regeneration in the early stage of reloading. Because we did not set an age-matched control group, we could not refute the possibility that recovery in MCSA, MND and myonuclear number involves muscle growth. MCSA in the 14 days of reloading group was significantly greater than that in the control group, but it was unclear whether MCSA in the 14 days of reloading group was as great as that of age-matched control. Therefore, in future study, we should use a model to consider the influence of growth.

The increase of mechanical stimuli and occurrence of myofiber damage were strongly related. Given the results of this study, there is a possibility that muscle hypertrophy occurred earlier than regeneration, so the difference of the degree of muscle damage with disuse muscle atrophy or reloading might influence hypertrophic reaction with reloading. If the extent of myofiber necrosis is inhibited, it is possible that hypertrophic reaction is facilitated more. Consequently, further studies are needed to characterize more definitively the importance of therapeutic procedure for an early stage of disuse muscle atrophy in order to analyze and examine how the differences in muscle damage influence the muscle hypertrophic process.

An important finding of the current study was that the processes of muscle hypertrophy and regeneration did not show uniform change. In addition, there were differences in the ratio of increase among the stages of hypertrophy and regeneration. Therefore, consideration of the duration and method of physiotherapeutic intervention for atrophied muscle on the basis of the process of hypertrophy and regeneration is needed to provide more effective physiotherapy. There is a possibility that muscle hypertrophy occurred earlier than regeneration, so it is thought that more efficient therapeutic procedures can be started with low loading for inhabitation of myofiber necrosis and promotion of hypertrophy. However, our results are insufficient to confirm this supposition, so further studies are needed.

\section{References}

1) Shirado O: Rehabilitation for musculoskeltal disorders in geriatric patients. Hokkaido Igaku Zasshi. 1997, 72: 389-396.

2) Sun B, Zhang LF, Gao F, Ma XW, Zhang ML, Liu J, Zhang LN, and Ma J: Daily short-period gravitation can prevent functional and structural changes in arteries of simulated microgravity rats. J Appl Physiol. 2004, 97: 1022-1031.

3) Williams PE: Effect of intermittent stretch on immobilised muscle. Ann Rheum Dis. 1988, 47: 1014-1016.

4) Sasa T, Sairyo K, Yoshida N, Fukunaga M, Koga K, Ishikawa $\mathrm{M}$, and Yasui N: Continuous muscle stretch prevents disuse muscle atrophy and deterioration of its oxidative capacity in rat tail-suspension models. Am J Phys Med Rehabil. 2004, 83: 851-856.

5) Mitchell PO, and Pavlath GK: A muscle precursor cell-de- 
pendent pathway contributes to muscle growth after atrophy. Am J Physiol Cell Physiol. 2001, 281: 1706-1715.

6) McCall GE, Allen DL, Linderman JK, Grindeland RE, Roy RR, Mukku VR, and Edgerton VR: Maintenance of myonuclear domain size in rat soleus after overload and growth hormone/IGF-I treatment. J Appl Physiol. 1998, 84: 14071412.

7) Wang XD, Kawano F, Matsuoka Y, Fukunaga K, Terada M, Sudoh M, Ishihara A, and Ohira Y: Mechanical load-dependent regulation of satellite cell and fiber size in rat soleus muscle. Am J Physiol Cell Physiol. 2006, 290: 981-989.

8) Hikida RS, Van Nostran S, Murray JD, Staron RS, Gordon SE, and Kraemer WJ: Myonuclear loss in atrophied soleus muscle fibers. Anat Rec. 1997, 247: 350-354.

9) Mozdziak PE, Pulvermacher PM, and Schultz E: Unloading of juvenile muscle results in a reduced muscle size $9 \mathrm{wk}$ after reloading. J Appl Physiol. 2000, 88: 158-164.

10) Mozdziak PE, Pulvermacher PM, and Schultz E: Muscle regeneration during hindlimb unloading results in a reduction in muscle size after reloading. J Appl Physiol. 2001, 91: 183-190.

11) Haida N: Cell science of disuse muscular atrophy. Rigaku ryohogaku. 1994, 21: 94-97. (Japanese)

12) Allen DL, Linderman JK, Roy RR, Grindeland RE, Mukku V, and Edgerton VR: Growth hormone/IGF-I and/or resistive exercise maintains myonuclear number in hindlimb unweighted muscles. J Appl Physiol. 1997, 83: 1857-1861.
13) Inoue $T$, Okita $M$, Takahasi $Y$, Harada $Y$, and Suzuki S: Effect of intermittent stretching on limitation of ankle joint mobility and disuse muscle atrophy in immobilized rat soleus muscle. Rigaku ryohogaku. 2007, 34: 1-9. (Japanese)

14) Yamazaki $T$ : Influence of hindlimb unweighting and intermittent weight bearing on dynamics of nuclei in rat soleus muscle. J Jpn Phys Ther Assoc. 2003, 6: 1-8.

15) Nonaka I : Muscle Pathology for the Clinician. $2^{\text {th }}$ ed, Tokyo, Japan Medical Journal Co., 1993, pp36-39.

16) Allen DL, Linderman JK, Roy RR, Bigbee AJ, Grindeland RE, Mukku V, and Edgerton VR: Apoptosis: a mechanism contributing to remodeling of skeletal muscle in response to hindlimb unweighting. Am J Physiol Cell Physiol. 1997, 273: 579-587.

17) Nguyen HX, and Tidball JG: Null mutation of gp91 $91^{\text {phox }}$ reduces muscle membrane lysis during muscle inflammation in mice. J Physiol. 2003, 553: 833-841.

18) Tidball JG: Inflammatory processes in muscle injury and repair. Am J Physiol Regul Comp Physiol. 2005, 288: 345353.

19) St Pierre BA, and Tidball JG: Differential response of macrophage subpopulations to soleus muscle reloading following rat hindlimb suspension. J Appl Physiol. 1994, 77: 290-297.

20) Tidball JG, and Wehling-Henricks M: Macrophages promote muscle membrane repair and muscle fiber growth and regeneration during modified muscle loading in mice in vivo. $\mathrm{J}$ Physiol. 2007, 578: 327-336. 